\title{
Metal-Supported Ultrathin Oxide Film Systems as Designable Catalysts and Catalyst Supports
}

\author{
Hans-Joachim Freund \\ Fritz-Haber-Institut der Max-Planck-Gesellschaft, \\ Faradayweg 4-6, 14195 Berlin, Germany
}

\begin{abstract}
Thin oxide films lend themselves as model supports for studies in heterogeneous catalysis, for example to study the growth and reaction of metal deposits (atoms, clusters, nanoparticles). If the thickness of the film is chosen appropriately these thin films are reasonable models to mimic the situation of bulk materials. If thin films below a critical thickness are studied these materials exhibit properties in their own right. Their structural properties may be tuned to control their functional characteristics. Possible implications for heterogeneous catalysis are discussed.
\end{abstract}

The ultimate goal of catalysis research is to design and tune activity and selectivity of catalysts by controlling their structural properties at the atomic level. Identification of concepts to achieve this goal is still one of the key issues of research in catalysis. A variety of different strategies have been put forward over the last decades [1, 2]. Most of these strategies are based on structure / reactivity relationships, taking different aspects of the exceedingly complex systems into account [3]. To this end model systems using metal particles supported on well ordered thin oxide films of appropriate thickness, which allow investigations with the rigor of modern surface science while grasping essential aspects of the complexity of real systems, have proven to give valuable insight into the details of geometric and electronic structure, as well as adsorption and reaction properties [4-9].

It has been recognized that the thickness of the oxide films, as they are grown on metal substrates, may be used as a design parameter to create materials of potential in catalysis. A concept to control the catalytic activity of a dispersed metal by the thickness of an insulating oxide layer was introduced some 20 years ago by Maier and co-workers using silica layers 
covering Pt [10-13]. In this case the catalytic performance for dehydrogenation of cycloalkenes depends significantly on the thickness of the silica films, which was explained by the decreasing transport of hydrogen atoms produced at the Pt surface through the silica film. The main experimental challenge, however, is to provide undisputable proof for the proposed mechanisms which is usually hampered either by the complexity of the samples investigated and/or the lack of appropriate methodology to exclude interference with alternative mechanisms. Developing experimental control together with realistic theoretical modeling, with respect to the thickness and structure of the oxide films [9], allows these structural properties to be used to control their functional characteristics and, thus, the catalytic properties of a metal deposited onto them. Here control may concern transport of species, e.g. hydrogen or oxygen, through the film or it may concern electronic interaction of the interior metal-oxide interface with adsorbed species on top. The latter idea is related to those proposed at the end of the 1940s by Cabrera and Mott in connection with metal oxidation [14]. In this case it was discussed whether electron tunneling through the growing oxide film during oxidation of a metal surface controls the oxidation rate via charge transfer towards the adsorbed species. On oxide surfaces charge transfer may also be induced via defects, such as color centres as recently theoretically predicted and experimentally proven $[15,16]$ or by reduced metal ions in the surface [17]. Charge transfer considerably modifies the chemical activity of the adsorbed species. For example, a charge transfer to carbon dioxide would lead to the formation of $\mathrm{CO}^{2-}$, a meta-stable radical ion which may dissociate or react with co-adsorbed species. Thus, a rather inert molecule would turn into a reactive species [18]. Similar effects have also been discussed for metal atoms and metal nano clusters [19] deposited onto charged oxide defects. Au, which is known to be chemically rather inert, may then be charged negatively, and according to calculations become chemically rather active, e.g. for carbon monoxide oxidation to carbon dioxide [20]. It has been claimed that this may be the reason for the activity of nanoscopic Au particles used as catalysts for oxidation reactions [21], although this connection has also been debated [22].

However, charging does not necessarily have to involve the presence of defects (as addressed in connection with the Mott-Cabrera mechanism for metal oxidation). On the basis of density functional theory (DFT) calculations it was recently proposed by Pacchioni and his coworkers that charge transfer may also occur for metal atoms (as opposed to oxygen in the case of the Cabrera-Mott mechanism) and metal clusters adsorbed on a supported thin oxide film, provided that the adsorbed metal exhibits a high electronegativity and the oxide film does not 
exceed a few mono-layers [15, 23]. In particular, Au atoms adsorbed on thin $\mathrm{MgO}(001)$ films grown on $\mathrm{Mo}(001)$ and $\mathrm{Ag}(001)$ are expected to be negatively charged in contrast to their counterparts on the corresponding bulk MgO (or thick MgO films) [15, 23]. In the latter case it was proven that Au atoms are essentially neutral [24]. Thus the thickness of the oxide film may serve as a parameter to tune the electronic properties of supported metals. Pacchioni and co-workers discussed the phenomenon in terms of the modification of the work function in the thin film system and the electronegativity (electron affinity) of the adsorbed species [25]. It is also connected to the ability of thin film to structurally relax upon charge transfer, i.e. the phenomenon contains a polaronic component stabilizing the charge transfer [23].

Work function changes have been extensively discussed for a variety of systems, including oxide-metal, alkali-halide-metal and metal-semiconductor interfaces [26-28]. The so-called Schottky model [29] has been used extensively to explain the alignment of the metal Fermi level with the valence and conductions bands of the semiconductor or insulator. The potential step involved in this level alignment on the one hand depends sensitively on the evanescent states in the gap. The formation of bonds at the interface may also induce an interfacial dipole moment due to the polarity of the bonds and the polarization of the metal electrons due to the presence of an ionic overlayer. This in turn leads to a change in the potential step at the metaloxide interfaces. Recently, it was demonstrated through modeling how different oxides may lead to drastic changes of the potential step [25]. The reasons for the predicted changes are very different, depending on the type of oxide.

Apparently, ionic oxides such as MgO cause a decrease of the potential step of metals such as Ag or Mo because the $\mathrm{O}^{2-}$ ions polarize the metal electrons away from the interface, effectively modifying the electron density and thus depleting the metal surface of electrons [28]. This effect strongly depends on the distance between the ionic layer and the top metal layer. In the case of more covalent oxides this effect is less strong. Here the oxygen ions still have some electron accepting ability leading to an accumulation of charge in the local metal substrate-oxygen bonds. This, in turn leads to an increase in potential step. $\mathrm{SiO}_{2}$ appears to be a good example for the latter case. In addition, there is an interesting trend observed in the theoretical predictions on the change of level alignment as the number of oxide layers increases: Except for the case of a single oxide layer, which establishes a special situation, there is little change in the level alignment as the film gets thicker then two layers [25]. However, as the film thickens it may loose its ability to structurally relax. 
With all this information in mind, it is foreseen, that by controlling the thickness of an oxide layer one has a unique opportunity to control charge transfer from the oxide-modified metal support to an adsorbate without using electrical control but by pure chemical means. If one could ensure stability under reaction conditions, thin oxide films on metallic supports would present a possibility to design model catalyst supports which control the charge state of an adsorbed species depending on their electronegativity of course, based on what has been discussed above, the oxide film cannot be thicker than the tunneling length. When growing oxide layers with thicknesses well exceeding this length, the relation of work function and electronegativity would still be favoring charge transfer to or from the adsorbed species but the electronic interaction would be shut off.

There is increasing evidence that the above scenario may hold true, and that it may, indeed be realized [30]. In an effort to confirm the predictions of Pacchioni and his co-workers from 2005 [15], Pd and Au adsorption has been studied on thick and thin $\mathrm{MgO}(100)$ layers while the morphology of the film was controlled via scanning tunneling microscopy and other means [30]. While Pd remains in the neutral state on both, thin and thicker substrates, Au has been shown to be neutral on the thicker film [24], while it charges negatively on a three layer thick film [30]. The charge state of Au on the thick film was measured in electron spin resonance experiments [24]. When Au is negatively charged on the thin MgO film, the distribution of atoms changes dramatically as revealed in figure 1 [30]. The Au atoms (figure 1a), due to their negative charge repel each other and built up a monolayer of low $\mathrm{Au}$ coverage with an average inter-atom distance of more than $1.5 \mathrm{~nm}$. Figure $1 \mathrm{~b}$ shows the distribution of Pd on a $\mathrm{MgO}(100)$ film of the same thickness. Most of the imaged protrusions are due to Pd atoms, very few larger aggregates containing a few atoms are also visible as slightly larger and brighter protrusions. The distribution of the Pd atoms is, in contrast to the $\mathrm{Au}$ atoms, typical for a statistical distribution of Pd atom distances [30] in a situation where the temperature is sufficiently low for long range diffusion not to occur. 

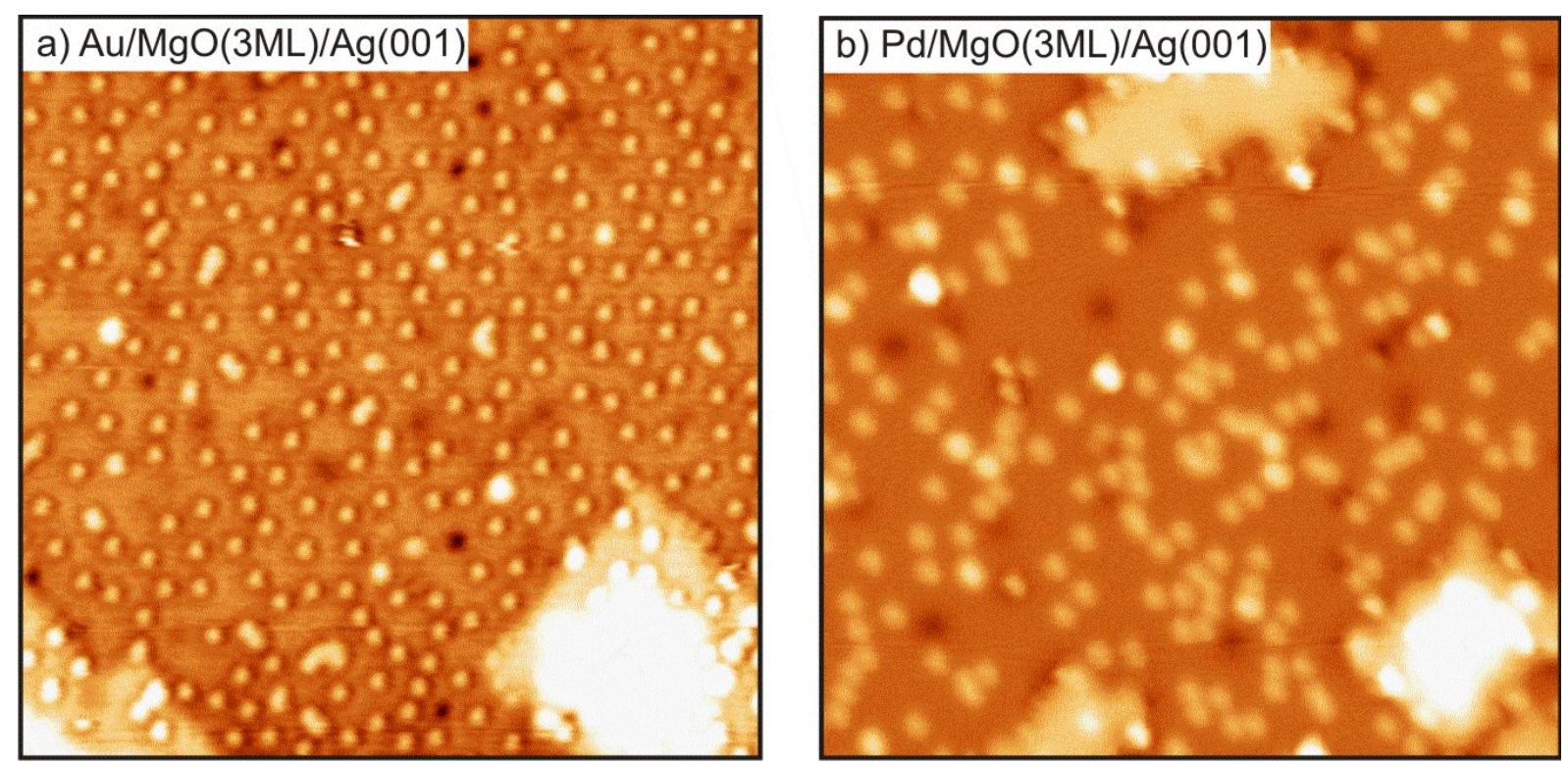

Figure 1: STM images $(30 \mathrm{~nm} \times 30 \mathrm{~nm})$ of (a) Au atoms adsorbed on $3 \mathrm{ML}$ thin $\mathrm{MgO}$ films $(\theta=2.9 \% \mathrm{ML} \mathrm{Au}$, $\left.\mathrm{V}_{\mathrm{S}}=+0.5 \mathrm{~V}, \mathrm{I}_{\mathrm{T}}=11 \mathrm{pA}\right)$; (b) Pd atoms adsorbed on $3 \mathrm{ML}$ thin MgO films $\left(\theta=2.3 \% \mathrm{ML} \mathrm{Pd}, \mathrm{V}_{\mathrm{S}}=+0.2 \mathrm{~V}, \mathrm{I}_{\mathrm{T}}=\right.$ $13 \mathrm{pA}$ ) [30].The images were taken at low temperature (4K). A flat $\mathrm{MgO}$ terrace is imaged with $\mathrm{Au}$ and $\mathrm{Pd}$ atoms (bright protrusions) distributed across the surface. MgO islands of thicker MgO films are imaged as brighter areas.

Had $\mathrm{Au}$ been deposited on a metallic surface, such as $\mathrm{Ag}(001)$, the distribution of $\mathrm{Au}$ atoms would have been very similar to that observed for Pd on all MgO substrates (not shown, see [30]). If $\mathrm{Au}$ is deposited on a thin alumina film, on the other hand, Au forms chains, whose length and orientation is determined by the interaction with the underlying $\mathrm{NiAl}(110)$ metallic alloy support (figure 2) [31]. 

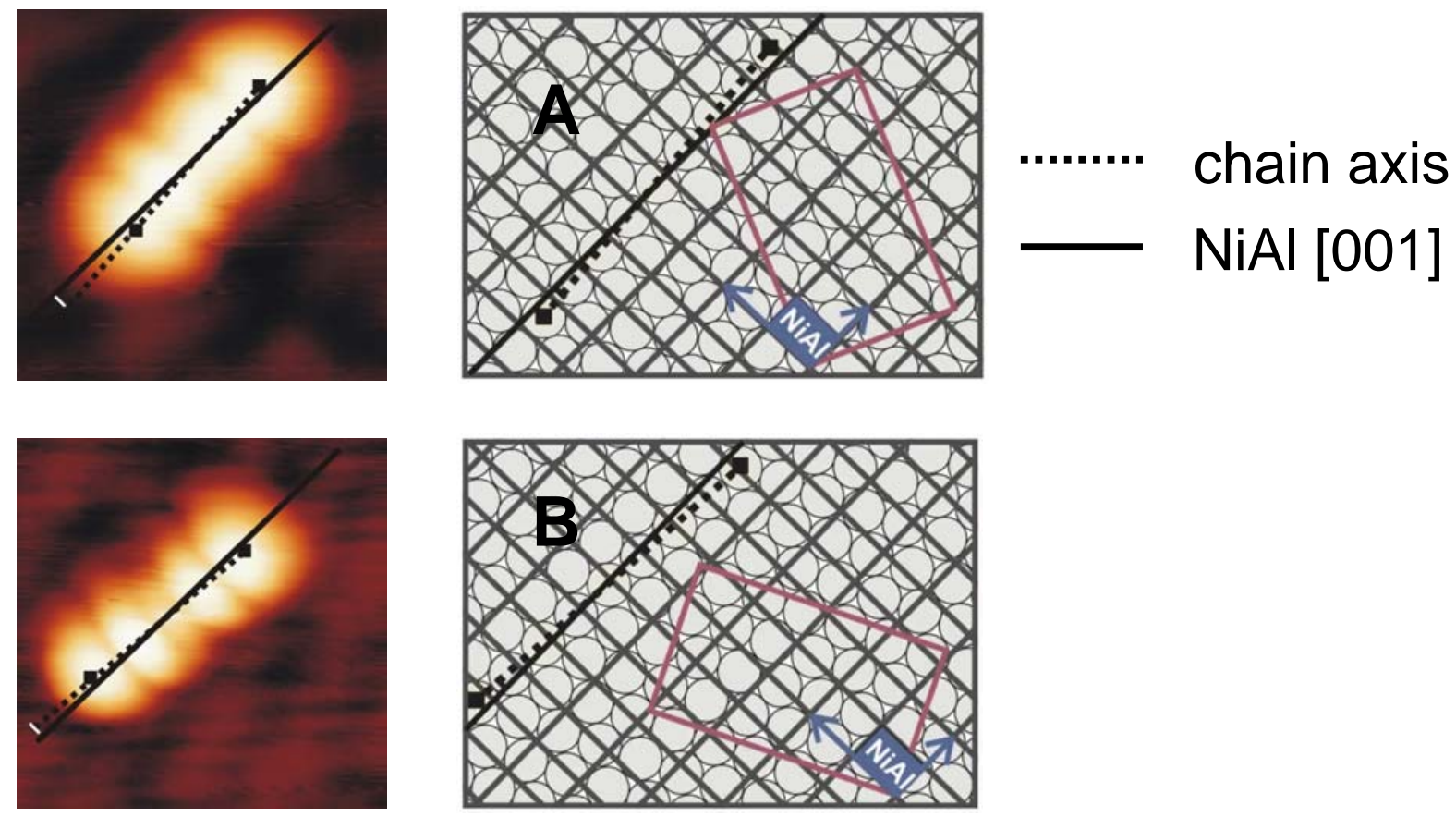

Figure 2: Au chains on alumina. The alumina films grow in two domains (A + B) on the $\mathrm{NiAl}(110)$ substrate. The orientation of the Au chains is determined by its interaction with the substrate, as indicated in the schematic representation in the panels on the right.

This exemplifies another important control parameter: The buried metal / oxide interface may be used to control the shape of the adsorbed species! Recently, Ricci et al. [32] predicted that $\mathrm{Au}$ nanoparticles change shape when they are deposited on a thin MgO film supported on a metal instead of a bulk MgO surface. Apparently, this change is induced by electrostatic interaction between the underlying metal and the metal-induced excess charge at the cluster oxide interface. This is accompanied with a crossover from three dimensional geometries to two-dimensional structures. These two-dimensional Au cluster structures have also been experimentally observed in the gas phase [33]. There is now experimental evidence that corroborates this prediction for MgO thin films [34]. Goodman and his group have recently reported the observation of the growth of two-dimensional Au structures (monolayers or bilayers) when grown on an ultrathin $\mathrm{TiO}_{2}$ overlayer on $\mathrm{Mo}(112)$ [35]. This system reveals extreme activity towards CO oxidation [35]. It is not unlikely that those results, which in the published literature [21,35] were not connected to the phenomena discussed in this Prospective, may, indeed, be induced by electronic interactions between the interior (underlying) Mo / oxide interface and the Au deposited from vacuum on top of the oxide.

There are more examples in the current literature, where the control of metal deposits by a metal / oxide interface has been discussed. 
$\mathrm{Au}$ atoms were deposited on a thin $\mathrm{FeO}(111)$ layer which was grown on top of a $\mathrm{Pt}(111)$ surface [36]. $\mathrm{FeO}(111)$ forms an overlayer with a large unit cell (so-called Moiré pattern) on top of $\mathrm{Pt}(111)$, so that only every $8^{\text {th }}$ oxygen atom in the rock-salt type $\mathrm{FeO}(111)$ layer coincides with a Pt atom in the substrate $[37,38]$. This induces a variation in the interfacial dipole which directs adsorbed Au atoms towards specific sites in the FeO layer [36, 39].

It was mentioned above that there is experimental evidence to the predicted two-dimensional growth of $\mathrm{Au}$ on a thin $\mathrm{MgO}$ film supported on another metal [34]. A similar growth mode was found for $\mathrm{Au}$ and $\mathrm{Pd}$ on $\mathrm{FeO}(111)$ thin films grown on Pt [36, 39, 40]. In this case twodimensional clusters are formed from neutral Pd atoms. The interaction between the interior metal oxide interface and the Pd clusters is so strong that the Pd switches to wet the Pt metal as the temperature is raised [41].

Very few attempts have been made so far to systematically explore adsorption and reaction of such systems. Basically, there are some studies on carbon monoxide adsorption indicating that the stretching frequencies change as expected based on the state of charge of the metal [34, 39, 40]. As far as reactivity is concerned, there are only the studies reported by Goodman and collaborators [21,35] on CO oxidation on the two-dimensional Au system supported on $\mathrm{TiO}_{2} / \mathrm{Mo}(112)$, which were mentioned above. There is a clear need for more such studies.

Metal supported ultrathin oxide film systems have designable properties as is quite obvious from the examples discussed above. Not all the design principles have been elucidated but a clear avenue for future studies is foreseen. It may be worthwhile to pursue these thoughts and even think of applications in catalysis. The approach may provide one route to model catalyst design that has not been followed systematically before.

There are lots of opportunities to explore various combinations of metals with varying electronegativities in combination with metal / oxide thin film systems. Furthermore, since it is often the interior metal / oxide interface that control the work function of the system, and thus its ability to donate or accept charge from/to the metal on top, one might think of ways to chemically modify this interface before the ultrathin oxide film is grown. If it turns out to be possible to retain the overall morphology of the system, such modifications would represent an interesting way to further explore the approach. Interfacial metal layers, which often wet a metal substrate, may be yet another interesting way forward. Also, modification of the underlying interface with alkali or halide adsorbates could be suitable, in particular, if one is 
interested in strong variations of work functions. Of course, it will be crucial from an experimental point of view to control all microscopic events at the interface or within the grown oxide layer.

In order to open up opportunities in applications, ultrathin oxide film systems need to be prepared on granular (powder) substrates. There are thin film oxide systems exhibiting self limiting growth. For example, alumina films grown on a NiAl alloy surface show such behavior [42]. In this respect, metal alloy systems, in general, could offer possibilities.

To conclude, there are opportunities for materials design, combining experiment and theory, that may be interesting and rewarding to explore. Just as in the early days of microelectronics fabrication, learning to tune band offsets at underlying interfaces by controlling interfacial dipoles may hold great promise for catalytic structures as well. 


\section{Acknowledgment}

I am very grateful to the coworkers mentioned in the references and in particular to Niklas Nilius, Thomas Risse and Martin Sterrer for their initiative and creativity related to the experimental work addressed in this prospective and Gianfranco Pacchioni for his deep theoretical insight and many fruitful discussions. I would like to thank the Deutsche Forschungsgemeinschaft and the Fonds der Chemischen Industrie for their support.

\section{References:}

[1] G. Ertl, H. Knözinger, J. Weitkamp (Eds.), Handbook of Heterogeneous Catalysis, Wiley-VCH Verlagsgesellschaft mbH, Weinheim, 1997.

[2] K. Honkala, A. Hellman, I.N. Remediakis, A. Logadottir, A. Carlsson, S. Dahl, C.H. Christensen, J.H. Nørskov, Science. 307 (2005) 555-558

[3] B.L. Kniep, T. Ressler, A. Rabis, F. Girgsdies, M. Baenitz, F. Steglich, R. Schlögl, Angew. Chem. Int. Ed. 43 (2004) 112-115.

[4] C.T. Campbell, Surf. Sci. Rep. 27 (1997) 1-111.

[5] C.R. Henry, Surf. Sci. Rep. 31 (1998) 231-326.

[6] H.-J. Freund, Angew. Chem. Int. Ed. Engl. 36 (1997) 452-475.

[7] H.-J. Freund, M. Bäumer, J. Libuda, T. Risse, G. Rupprechter, S. Shaikhutdinov, J. Catal. 216 (2003) 223.

[8] D.W. Goodman, J. Cat. 216 (2003) 213-222.

[9] H. Freund, D.W. Goodman, in: G. Ertl, H. Knözinger, F. Schüth, J. Weitkamp (Eds.), Handbook of Heterogeneous Catalysis, Second ed., Wiley-VCH Verlagsgesellschaft mbH, Weinheim, 2007.

[10] A.B. McEwen, W.F. Maier, R.H. Fleming, S.M. Baumann, Nature. 329 (1987) 531.

[11] A.B. McEwen, W.F. Maier, D.A. Smith, in: J.W. Ward (Ed.), Catalysis 1987 Elsevier Science Publishers B.V., Amsterdam, 1987, p. 823.

[12] R.H. Fleming, S.M. Baumann, G.P. Meeker, W.F. Maier, in: A. Benninghoven (Ed.), Secondary Ion Mass Spectrometry: SIMS VI, Wiley, Paris, 1987, p. 585.

[13] J.M. Cogen, K. Ezaz-Nikpay, R.H. Fleming, S.M. Baumann, W.F. Maier, Angew. Chem. Int. Ed. 26 (1987) 1182-1184.

[14] N. Cabrera, N.F. Mott, Rep. Progr. Phys. 12 (1948) 163.

[15] G. Pacchioni, L. Giordano, M. Baistrocchi, Phys. Rev. Lett. 94 (2005) 226104.

[16] M. Sterrer, M. Yulikov, E. Fischbach, M. Heyde, H.P. Rust, G. Pacchioni, T. Risse, H.-J. Freund, Angew. Chem. Int. Ed. 45 (2006) 2630-2632.

[17] O. Seiferth, K. Wolter, B. Dillmann, G. Klivenyi, H.-J. Freund, D. Scarano, A. Zecchina, Surf. Sci. 421 (1999) 176-190.

[18] H.-J. Freund, M.W. Roberts, Surf. Sci. Rep. 25 (1996) 225-273.

[19] S. Abbet, E. Riedo, H. Brune, U. Heiz, A.M. Ferrari, L. Giordano, G. Pacchioni, J. Am. Chem. Soc. 123 (2001) 6172-6178.

[20] B. Yoon, H. Häkkinen, U. Landman, A. Wörz, J.-M. Antonietti, S. Abbet, K. Judai, U. Heiz, Science. 307 (2005) 403.

[21] M. Valden, X. Lai, D.W. Goodman, Science. 281 (1998) 1647-1650

[22] M. Haruta, Cattech. 6 (2002) 102-115.

[23] L. Giordano, G. Pacchioni, Phys. Chem. Chem. Phys. 8 (2006) 3335 - 3341. 
[24] M. Yulikov, M. Sterrer, M. Heyde, H.P. Rust, T. Risse, H.-J. Freund, G. Pacchioni, A. Scagnelli, Phys. Rev. Lett. 96 (2006) 146804.

[25] L. Giordano, F. Cinquini, G. Pacchioni, Phys. Rev. B. 73 (2006) 045414.

[26] W. Mönch, Rep. Progr. Phys. 53 (1990) 221.

[27] C. Noguera, Physics and Chemistry at Oxide Surfaces, Cambridge University Press, Cambridge, 1995.

[28] J. Goniakowski, C. Noguera, Interface Sci. 12 (2004) 93-103.

[29] W. Schottky, Phys. Z. 41 (1940) 570-573.

[30] M. Sterrer, T. Risse, U. Martinez, L. Giordano, M. Heyde, H.P. Rust, G. Pacchioni, H.-J. Freund, Phys. Rev. Lett. submitted (2006).

[31] M. Kulawik, N. Nilius, H.J. Freund, Phys. Rev. Lett. 96 (2006) 036103.

[32] D. Ricci, A. Bongiorno, G. Pacchioni, U. Landman, Phys. Rev. Lett. 97 (2006) 036106.

[33] F. Furche, R. Ahlrichs, P. Weis, C. Jacob, S. Gilb, T. Bierweiler, M.M. Kappes, J. Chem. Phys. 117 (2002) 6982-6990.

[34] M. Sterrer, T. Risse, M. Heyde, N. Nilius, H.P. Rust, H.-J. Freund, Phys. Rev. Lett. submitted.

[35] M.S. Chen, D.W. Goodman, Science. 306 (2004) 252-255.

[36] N. Nilius, E.D.L. Rienks, H.-P. Rust, H.-J. Freund, Phys. Rev. Lett. 95 (2005) 066101.

[37] M. Ritter, W. Ranke, W. Weiss, Phys. Rev. B. 57 (1998) 7240-7251.

[38] W. Ranke, M. Ritter, W. Weiss, Phys. Rev. B. 60 (1999) 1527.

[39] E.D.L. Rienks, N. Nilius, H. Freund, to be published.

[40] S. Shaikhutdinov, R. Meyer, D. Lahav, M. Bäumer, T. Klüner, H.J. Freund, Phys. Rev. Lett. 91 (2003) 076102.

[41] R. Meyer, D. Lahav, T. Schalow, M. Laurin, B. Brandt, S. Schauermann, S. Guimond, T. Klüner, H. Kuhlenbeck, J. Libuda, Surf. Sci. 586 (2005) 174.

[42] R.M. Jaeger, H. Kuhlenbeck, H.-J. Freund, M. Wuttig, W. Hoffmann, R. Franchy, H. Ibach, Surf. Sci. 259 (1991) 235. 